\title{
GENETIC POTENTIAL AND ASSOCIATION AMONG MORPHO- PHYSIOLOGICAL TRAITS OF PETUNIA INBRED LINES
}

\author{
MAHMOOD, A. ${ }^{1}-$ ALI, Q $.{ }^{2,6^{*}}-$ AHMAD, S. ${ }^{1}$ - BAKHSH, A. ${ }^{3}$ - MAHPARA, S. ${ }^{3}-$ KAMARAN, ${ }^{3}{ }^{3}-$ \\ MAMOON-UR-RASHID, M. ${ }^{4}$ - SALMAN, S. ${ }^{5}$ - WASEEM, M. ${ }^{6}$ - HAIDER, M. S. ${ }^{1}$ - NASIR, I. A. ${ }^{7}$ \\ ${ }^{1}$ Institute of Agricultural Sciences, University of the Punjab Lahore, Lahore, Pakistan \\ ${ }^{2}$ Institute of Molecular Biology and Biotechnology, University of Lahore, Lahore, Pakistan \\ ${ }^{3}$ Department of Plant Breeding and Genetics, Ghazi University, Dera Ghazi Khan, Pakistan \\ ${ }^{4}$ Department of Entomology, Faculty of Agriculture, Gomal University, Dera Ismail Khan, \\ Pakistan
}

${ }^{5}$ Department of Plant Breeding and Genetics, Gomal University, Dera Ismail Khan, Pakistan

${ }^{6}$ Department of Agronomy, Faculty Agriculture, Lasbela University of Agriculture, Water and Marine Science, Uthal, Lasbela, Pakistan

${ }^{7}$ Centre of Excellence in Molecular Biology, University of the Punjab Lahore, Lahore, Pakistan

*Corresponding author

e-mail:saim1692@gmail.com

(Received $7^{\text {th }}$ Feb 2019; accepted $8^{\text {th }}$ Apr 2019)

\begin{abstract}
Petunia hybrida is considered as an important and beautiful ornamental flowering plant, grown throughout the world for its beauty and attractiveness. Various petunia hybrids have been produced by different countries. The present study was carried out to investigate the higher seed yield petunia line developed through selfing to produce $F_{1}$ hybrids during the experimental seasons of the 2011-2014 period. The lines IAGS-P8, IAGS-P9 and IAGS-P11 performed well, both in the cluster analysis and minimum spanning tree, the inbred lines IAGS-P8 and IAGS-P9 showed to a higher similarity percentage. From statistical and regression analysis showed that stomata conductance, chlorophyll 'a' contents, chlorophyll ' $b$ ' contents, flowers per plant, flower fresh weight, seed area, seed weight and abscisic acid contributed higher to seed yield per plant in petunia. The higher abscisic acid concentration suggested that the petunia genotypes may be used to develop better seed yields and higher number of flowers per plant in petunia under environmental stress. This method proved to be more efficient as it reduced the cost, time and efficacy for better selection in petunia improvement program. Still, further studies are required which should cover different years and locations.
\end{abstract}

Keywords: Petunia hybrida, abscisic acid, multivariate analysis, heritability, genetic advance

\section{Introduction}

Petunia hybrida is an important and the mostly used ornamental plant throughout the world from several years as commercially grown ornamental plant. Petunia hybrida is an important and mostly used genera to develop Petunia new varieties and hybrids. Griesbach (2007) has reported that Petunia has an old history in sense of development as an ornamental plant as compared with new emerging ornamental crops plant species. Ganga et al. (2011) suggested that the production of hybrid seed has been adapted by various ornamental crop plants breeding program to develop new colour and varieties of ornamental plants. It has been found that there are 20 South American origin species of 
Petunia (Selaru, 2008), 14 herbaceous species (Toma, 2009). Selaru (2008) reported that the specie Petunia hybrida Hort. (P. axillaris Lam. $\times$ P. violacea Lindl.) has been presented with higher decorative value. It has been confirmed from findings of various researchers that the 14 familiar species are consisted of genus Petunia (Stehmann et al., 2009). The Petunia hybrida Hort. as an ornamental plant species, used as ornamental plant in loans, fields and gardens for its abundant flowering ability (Toma et al., 2011). Dole and Wilkins (1999) reported that most of the Petunia species have been developed in Brazil, Uruguay or Argentina. In modern era a large number of cultivars have been developed in the form of varieties, hybrids and cultivars with variety of colors and patterns. Depending on flower size, color and habit, four groups of Petunias have been defined as, multiflora, milliflora, grandiflora, ground cover or spreading. Even every year new varieties of Petunia have been developed by ornamental breeders (Toma, 2009).

Bender (2006) found that the new and modern species of petunias are hybrids in nature like garden petunia (Petunia axillaris) or ambrosial white and violet petunia ( $P$. integrifolia, and $P$. violacea). The old or wild type petunia species, varieties or hybrids use has been less down now a day. Petunia is grown throughout the world for its beauty, higher growth rate, different color ranges and continued flowering up to about 6 months of plant life cycle. It blossoms most abundantly under hot summers conditions (Bala, 2012; Selaru, 2008). Erwin (2006) found that the higher blooming ability, petunia is even grown in baskets, landscaping by the forms, in alloyed trays on roads and even in room open spaces (Erwin, 2006). The present study was conducted to develop inbred lines of petunia through selfing at growing seasons. The data of various morphological, physiological and seed yield traits was recorded to access the performance of inbred lines under development. The identification of promising inbred lines was also done.

\section{Material and methods}

The prescribed study was carried out in the research area of Institute of Agricultural Sciences, University of the Punjab Lahore, Pakistan located at $31.4779^{\circ} \mathrm{N}, 74.2623^{\circ} \mathrm{E}$ with average higher temperature of about $37^{\circ} \mathrm{C}$ with highest up to $45^{\circ} \mathrm{C}$ during summer season, $11^{\circ} \mathrm{C}$ with lowest $3{ }^{\circ} \mathrm{C}$ during winter season. Twelve petunia lines, IAGS-P1, IAGS-P2, IAGS-P3, IAGS-P4, IAGS-P5, IAGS-P6, IAGS-P7, IAGS-P8, IAGS-P9, IAGS-P10, IAGS-P11 and IAGS-P12 were selected and grown in the field during 2014, selfing of all the lines was carried out for three successive growing seasons (2011-13) to develop inbred lines, the selfed seed were collected to develop next generation (Figs. 1 and 2). The selfed seed was grown in three replication under completely randomized block design during growing season of 2014, and data for various traits of 10 selected plants from each of three replications was recorded during all three growing seasons and average of data was used for results and interpretation, the traits were included photosynthetic rate (A), leaf temperature (LT), transpiration rate (E), stomata conductance (gs) and Sub-stomata $\mathrm{CO}_{2}$ Concentration (Ci) through the use of IRGA (Infrared Gas Analyzer, Model: LI-6400XT), chlorophyll 'a' content (Chl. a), chlorophyll ' $b$ ' content (Chl. b) in fresh matter was measured using the dimethyl sulfoxide extraction method (Hiscox and Israelstam, 1978), leaves per plant (LPP), plant height (PH), stem diameter (SD), flowers per plant (FPP), leaf length (LL), leaf width (LW), leaf area (LA), fresh leaf weight (FLW), fresh stem weight (FSW), flower 
weight (FW), seeds per fruit (SPF), 100-seed weight (HSW), seed area (SA), capsule weight (CW), abscisic acid (ABA) by using HPLC method (Seo and Koshiba, 2002), and seed yield per plant (SYP) were collected. Water use efficiency (WUE) was recorded through using given formula:

$$
\text { Water use efficiency }(\%)=\frac{\text { Transpiration rate }}{\text { Photosynthetic rate }} \times 100
$$

The collected data was statistically analyzed by using analysis of variance technique, principal component analysis, correlation analysis, regression analysis, factor analysis, spinning tree and cluster analysis (Steel et al., 1997) through using GenStat 18 (SP1) version.
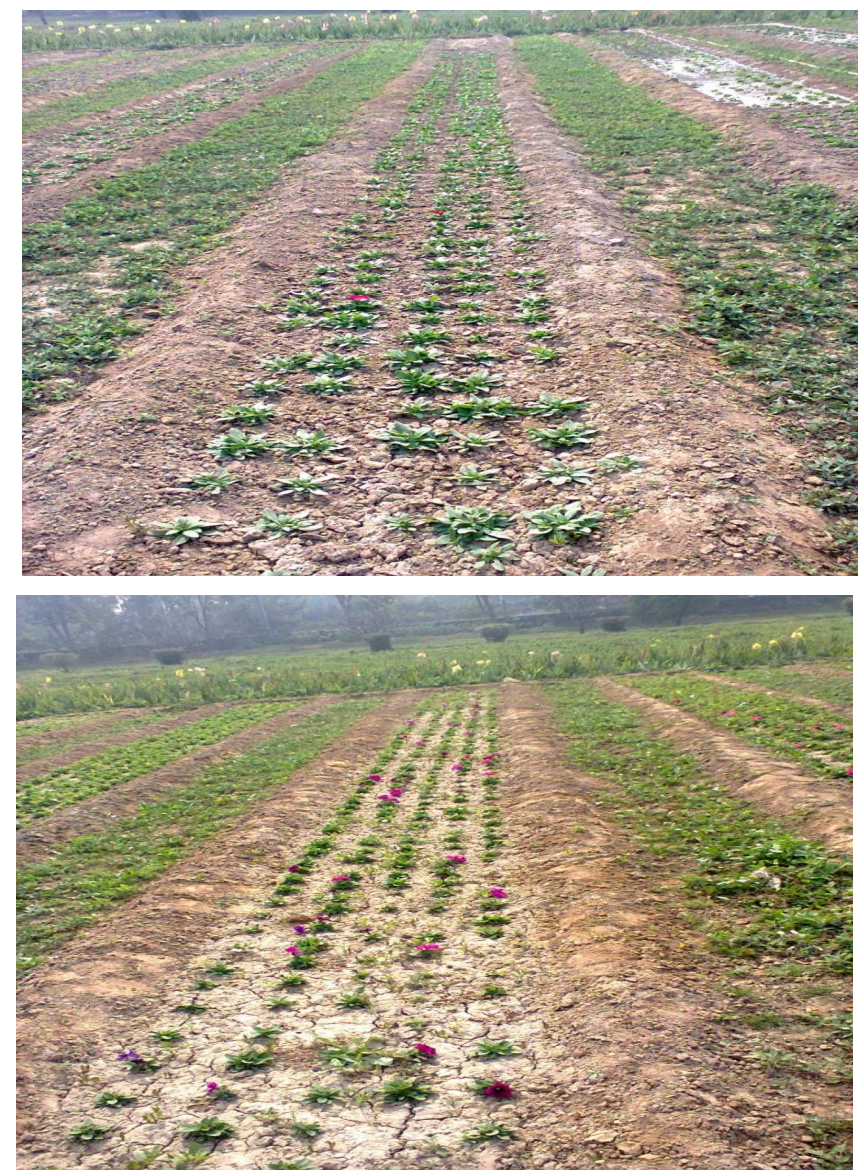

Figure 1. Twelve lines petunia hybrida grown in filed

\section{Heritability estimate}

Broad sense heritability was calculated by using genotypic, phenotypic and environmental variances according to Burton (1951):

$$
\text { Broad sense heritability }=\frac{\left(\partial_{g}^{2}+\partial_{e}^{2}\right)-\partial_{e}^{2}}{\partial_{g}^{2}+\partial_{e}^{2}} \times 100
$$


$\partial^{2} \mathrm{~g}=$ genotypic variance, $\partial^{2}{ }_{\mathrm{e}}=$ environmental variance, $\partial^{2}{ }_{\mathrm{g}}+\partial^{2}{ }_{\mathrm{e}}=$ phenotypic variance, $\left(\partial_{\mathrm{g}}^{2}+\partial_{\mathrm{e}}^{2}\right)-\partial_{\mathrm{e}}^{2}=$ genotypic variance.

Genetic advance (GA) was calculated by the following formula (Falconer, 1989):

$$
\mathrm{GA}=\sigma_{p} x h^{2} x i
$$

where: $\sigma_{\mathrm{p}}=$ the phenotypic standard deviation; $\mathrm{h}^{2}=$ estimate of broad sense heritability; $\mathrm{i}=$ constant value $(1.755)$ that reflects selection intensity $(10 \%)$.

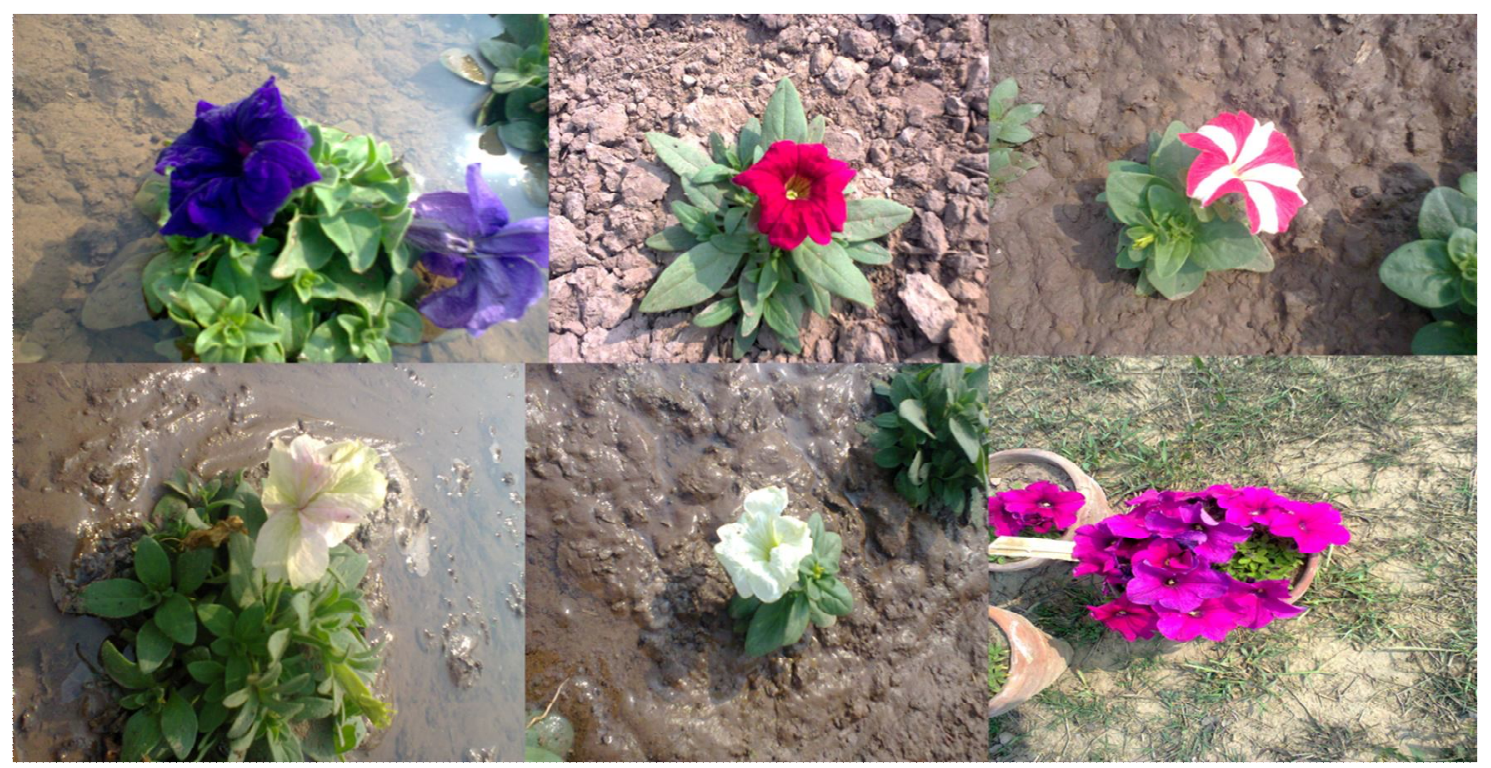

Figure 2. Close view of different petunia hybrid in field and pot (for transplanting in field)

\section{Results and discussions}

It was persuaded from the results (Table 1) that significant differences were found for all studied traits. Broad sense heritability was found higher for photosynthetic rate $(90.825 \%)$, stomata conductance $(80.268 \%)$, sub-stomata $\mathrm{CO}_{2}$ concentration $(96.035 \%)$, water use efficiency $(89.847 \%)$, leaves per plant $(98.750 \%)$, plant height $(92.613 \%)$, leaf length $(83.584 \%)$, flowers per plant $(92.911 \%)$, leaf area $(84.460 \%)$, fresh stem weight $(85.266 \%)$, seeds per fruit $(96.379 \%)$ and abscisic acid $(85.205 \%)$ while lower for chlorophyll b (23.527\%), leaf width (37.084\%), fresh leaf weight (24.074\%), flower weight $(26.145 \%)$, seed area $(26.781 \%)$ and seed yield per plant $(4.359 \%)$. Genetic advance was found higher for all of the studied traits seeds per fruit. Higher heritability suggested that the selection of petunia lines on the basis of these traits for the development of hybrids may be fruitful. Various researchers while working on different crop plants have described about higher heritability for these traits as reported in our study (Ali et al., 2013, 2014; Fawad et al., 2017). Higher genetic advance suggested that the selection of lines may also be helpful to develop synthetic varieties. The similar findings for different crops have been reported by various researchers (Ali et al., 2013, 2016). It was found from results (see Appendix) that the line IAGS-P8, IAGS-P9 and IAGS-P11 performed better than all other lines. Correlation analysis provides an opportunity to the research to select genotypes of crop plant to improve crop plant growth and production. In our study, significant correlation (Table 2) was found for 
photosynthetic rate with chlorophyll ' $\mathrm{a}$ ' contents, sub-stomata $\mathrm{CO}_{2}$ concentration, water use efficiency, plant height, flowers per plant, leaf width and abscisic acid. Abscisic acid was found to be significantly correlated with most of the studied traits including seed yield per plant. Stomata conductance showed also significant correlation with transpiration rate, water use efficiency, flowers per plant, leaf area, fresh shoot weight, seeds per fruit, abscisic acid and leaf width. Seed yield per plant was significantly correlated with photosynthetic rate, leaf temperature, leaves per plant, stem diameter, seed weight and abscisic acid. The positive and significant correlation suggested that the selection of lines to develop hybrids and synthetic varieties may be helpful to improve the growth and development of petunia, the significant correlation of abscisic acid with morphological, seed yield and physiological traits indicated that the selection of petunia lines on the basis of good abscisic acid production may be fruitful to improve drought tolerance in petunia (Mahmood et al., 2017; Sah et al., 2016; Skubacz et al., 2016; Zao et al., 2016; Saradadevi et al., 2017). Kaczperski et al. (1991) estimated growth of the petunia and development in combined effects of light and temperatures. The adverse effect of an optimum temperature environment for minimum time to petunia hybrida was begin to be $25{ }^{\circ} \mathrm{C}$, and a minimum circadian light intensity for adequate growth was begin to be 13 molm $^{-2} \mathrm{~d}^{-1}$. The adeptness for petunia to growth and development with increase in light and temperature has afresh been appeared, so there is befalling to optimize development, crop plant growth, crop scheduling and higher with ecology changes in light and increase in temperature. However, the accessible delay of growth and development with top abundance and the access of $\mathrm{CO}_{2}$ in an optimized environment showed not extensively have adverse effects (Ahmad and Tahir, 2017; Blanchard and Runkle, 2009; Mustafa et al., 2017).

The stepwise regression (Table 3) was performed to find out the traits that were highly contributing to the seed yield per plant of petunia, from results it was predicted that stomata conductance (120.311), chlorophyll ' $a$ ' contents (2.775), chlorophyll ' $b$ ' contents (2.1040, flowers per plant (2.484), flower fresh weight (11.364), seed area (7.062), seed weight (31.497) and abscisic acid (15.356) contributed higher to seed yield per plant in petunia but it could be biased as previous literature also reported the error effect of stepwise regression (El-Badaway and Mehasen, 2011; Ahmad et al.; 2012, 2016) while handling a large number of independent variables. The Intercept $=128.387, \mathrm{R}^{2}=0.832$, Adjust $\mathrm{R}^{2}=0.276$ and Standard Error $=0.728$ was found with expected regression equation as follows:

$$
\begin{aligned}
Y & =128.387+\left(0.224 X_{1}\right)+\left(-0.235 X_{2}\right)+\left(2.775 X_{3}\right)+\left(2.2 .104 X_{4}\right)+\left(120.311 X_{5}\right) \\
& +\left(-4.418 X_{6}\right)+\left(-0.007 X_{7}\right)+\left(-0.070 X_{8}\right)+\left(-0.252 X_{9}\right)+\left(0.116 X_{10}\right)+\left(0.004 X_{11}\right) \\
& +\left(2.484 X_{12}\right)+\left(-0.115 X_{13}\right)+\left(-17.234 X_{14}\right)+\left(-80.073 X_{15}\right)+\left(11.364 X_{16}\right)+\left(-10.121 X_{17}\right) \\
& +\left(0.068 X_{18}\right)+\left(-12.101 X_{19}\right)+\left(-0.033 X_{20}\right)+\left(7.062 X_{21}\right)+\left(31.497 X_{22}\right)+\left(15.356 X_{23}\right)
\end{aligned}
$$

Various researchers have used PCA (Principal Component Analysis) to evaluate the effects of independent traits (Table 4) in large number for selecting the fruitful variability percentage from a large population (Fawad et al., 2017; Ali et al., 2014). It has been reported from study conducted by Greenacre (2010) that the eigenvalues (used in PCA) shows the primary importance of numerical diagnostics for assessing the variation which a number of large traits/variables contributed for the dependent structure also to display the data matrix in a graphical form. 
Table 1. Genetic components for various morpho-physiology and yield traits of petunia

\begin{tabular}{|c|c|c|c|c|c|c|c|c|c|c|}
\hline Traits & M.S & G.M $\pm \mathbf{S E}$ & GV & GCV \% & PV & PCV \% & EV & ECV \% & $h^{2} b s \%$ & GA\% \\
\hline Photosynthetic rate $\left(\mu \mathrm{gCO}_{2} \mathrm{~s}^{-1}\right)$ & $124.356^{*}$ & $15.114 \pm 0.0094$ & 40.102 & 168.621 & 44.153 & 176.933 & 4.051 & 53.593 & 90.825 & 75.097 \\
\hline Leaf temperature $\left({ }^{\circ} \mathrm{C}\right)$ & $139.563 *$ & $22.184 \pm 0.0613$ & 38.703 & 135.136 & 62.157 & 171.255 & 23.454 & 105.198 & 62.267 & 40.651 \\
\hline Chlorphyll a (mg g ${ }^{-1}$ fr. wt.) & $13.467^{*}$ & $3.219 \pm 0.0579$ & 4.074 & 112.848 & 5.319 & 128.943 & 1.245 & 62.383 & 76.593 & 96.905 \\
\hline Chlorphyll b (mg g-1 fr. wt.) & $17.972 *$ & $1.268 \pm 0.0386$ & 2.875 & 135.008 & 12.221 & 278.339 & 9.346 & 243.404 & 23.527 & 91.503 \\
\hline Stomata conductance $\left(\mathrm{mmol} \mathrm{m}^{-2} \mathrm{~s}^{-1}\right)$ & $1.294^{*}$ & $0.042 \pm 0.0068$ & 0.399 & 361.210 & 0.497 & 403.169 & 0.098 & 179.089 & 80.268 & 324.911 \\
\hline Transpiration rate $\left(\mathrm{mm}\right.$ day $\left.^{-1}\right)$ & $1.029 *$ & $0.914 \pm 0.0176$ & 0.272 & 55.482 & 0.485 & 74.087 & 0.213 & 49.097 & 56.082 & 77.574 \\
\hline Sub-stomata $\mathrm{CO}_{2}$ concentration $\left(\mu \mathrm{mol} \mathrm{mol}{ }^{-1} \mathrm{CO}_{2}\right)$ & $238.323^{*}$ & $149.119 \pm 0.8165$ & 78.363 & 72.548 & 81.598 & 74.030 & 3.235 & 14.740 & 96.035 & 10.226 \\
\hline Water use efficiency $(\%)$ & $37.356^{*}$ & $7.781 \pm 0.1471$ & 12.000 & 132.920 & 13.356 & 140.229 & 1.356 & 44.682 & 89.847 & 84.844 \\
\hline Leaves per plant & $234.974 *$ & $87.742 \pm 0.4714$ & 77.996 & 94.820 & 78.983 & 95.418 & 0.987 & 10.669 & 98.750 & 17.755 \\
\hline Plant height $(\mathrm{cm})$ & $209.803 *$ & $53.719 \pm 0.0910$ & 68.123 & 110.474 & 73.557 & 114.795 & 5.434 & 31.201 & 92.613 & 24.974 \\
\hline Stem diameter $(\mathrm{cm})$ & $1.073^{*}$ & $0.532 \pm 0.0012$ & 0.311 & 78.057 & 0.450 & 93.879 & 0.139 & 52.157 & 69.134 & 159.345 \\
\hline Flowers per plant & $398.244^{*}$ & $147.16 \pm 0.4714$ & 129.456 & 95.819 & 139.333 & 99.407 & 9.877 & 26.467 & 92.911 & 13.651 \\
\hline Leaf length $(\mathrm{cm})$ & $36.357^{*}$ & $7.121 \pm 0.0216$ & 11.374 & 134.112 & 13.608 & 146.692 & 2.234 & 59.435 & 83.584 & 85.567 \\
\hline Leaf width (cm) & $5.459^{*}$ & $1.618 \pm 0.0294$ & 1.162 & 92.533 & 3.134 & 151.951 & 1.972 & 120.527 & 37.084 & 84.878 \\
\hline Leaf Area $\left(\mathrm{cm}^{2}\right)$ & $39.023 *$ & $6.124 \pm 0.1392$ & 12.256 & 138.894 & 14.511 & 151.133 & 2.255 & 59.578 & 84.460 & 88.879 \\
\hline Fresh leaf weight (g) & $3.214^{*}$ & $0.661 \pm 0.0249$ & 0.522 & 89.340 & 2.169 & 182.086 & 1.647 & 158.662 & 24.074 & 95.103 \\
\hline Fresh stem weight (g) & $204.224 *$ & $46.321 \pm 0.0535$ & 64.367 & 114.352 & 75.490 & 123.838 & 11.123 & 47.536 & 85.266 & 26.413 \\
\hline Flower weight (g) & $2.029^{*}$ & $0.636 \pm 0.0205$ & 0.348 & 76.110 & 1.332 & 144.736 & 0.984 & 124.385 & 26.145 & 98.045 \\
\hline Seeds per fruit & $998.267^{*}$ & $869.169 \pm 0.4714$ & 328.640 & 61.597 & 340.986 & 62.743 & 12.346 & 11.939 & 96.379 & 3.606 \\
\hline 100-seed weight (mg) & $65.324^{*}$ & $13.131 \pm 0.0082$ & 17.363 & 120.043 & 30.597 & 159.354 & 13.234 & 104.801 & 56.748 & 45.721 \\
\hline Seed area $(\mathrm{mm})$ & $2.091 *$ & $0.418 \pm 0.0052$ & 0.365 & 101.044 & 1.362 & 195.254 & 0.997 & 167.075 & 26.781 & 153.556 \\
\hline Seed yield per plant & $1.129 *$ & $52.213 \pm 0.0294$ & 0.099 & 4.354 & 1.092 & 14.462 & 0.993 & 13.791 & 9.066 & 5.769 \\
\hline Capsule weight (mg) & $98.672 *$ & $115.121 \pm 0.0618$ & 25.447 & 47.015 & 47.779 & 64.423 & 22.332 & 44.044 & 53.259 & 62.284 \\
\hline Abscisic acid (mg/100 g fresh leaf weigh) & $572.897^{*}$ & $127.126 \pm 0.0163$ & 180.517 & 125.221 & 211.862 & 135.658 & 31.345 & 52.180 & 85.205 & 18.906 \\
\hline
\end{tabular}

*Significant at 5\% probability level. Mean Sum of Squares (M.S), Grand mean (G.M), Genotypic variance (GV), Genotypic coefficient of variance (GCV \%), Phenotypic variance (PV), Phenotypic coefficient of variance (PCV \%), Environmental Variance (EV), Environmental coefficient of variance (ECV \%), Broad sense heritability (h2bs \%), Genetic advance (GA) 
Table 2. Correlation among various morpho-physiology and yield traits of petunia

\begin{tabular}{|c|c|c|c|c|c|c|c|c|c|c|c|c|c|c|c|c|c|c|c|c|c|c|c|}
\hline Traits & A & LT & Chl. a & Chl. b & gs & E & $\mathrm{Ci}$ & WUE & LPP & PH & SD & FPP & LL & LW & LA & FLW & FSW & FW & SPF & HSW & SA & SW & $\mathrm{ABA}$ \\
\hline LT & -0.1013 & & & & & & & & & & & & & & & & & & & & & & \\
\hline Chl. a & 0.8128* & -0.0851 & & & & & & & & & & & & & & & & & & & & & \\
\hline Chl. b & -0.0597 & -0.2476 & -0.0181 & & & & & & & & & & & & & & & & & & & & \\
\hline gs & -0.2867 & 0.1704 & -0.1030 & $0.4668^{*}$ & & & & & & & & & & & & & & & & & & & \\
\hline E & -0.0079 & $0.4530^{*}$ & $-0.0727 \mid$ & $0.8146^{*}$ & $0.4579 *$ & & & & & & & & & & & & & & & & & & \\
\hline $\mathrm{Ci}$ & $0.3950^{*}$ & -0.2423 & $0.3196 *$ & 0.0541 & 0.3187 & 0.0697 & & & & & & & & & & & & & & & & & \\
\hline WUE & $0.5112 *$ & -0.2758 & $0.3659 *$ & $0.7206^{*}$ & $0.4996 *$ & $0.8432^{*}$ & 0.2287 & & & & & & & & & & & & & & & & \\
\hline LPP & 0.2184 & 0.1949 & $-0.1063 \mid$ & -0.2864 & -0.1038 & $-0.6049 *$ & -0.2114 & $-0.6657^{*}$ & & & & & & & & & & & & & & & \\
\hline PH & $0.4889^{*}$ & 0.0884 & $0.5840^{*}$ & $-0.3759^{*}$ & $-0.2407 \mid$ & $-0.3948 *$ & $0.5473 *$ & -0.0364 & -0.2126 & & & & & & & & & & & & & & \\
\hline SD & -0.0771 & 0.2057 & 0.2103 & -0.1540 & -0.1632 & $-0.4087 *$ & 0.1115 & -0.2716 & 0.0482 & $0.5810^{*}$ & & & & & & & & & & & & & \\
\hline FPP & $0.5809^{*}$ & -0.3171 & $0.4399 *$ & $-0.3461^{*}$ & $0.6668 *$ & -0.2286 & -0.1656 & $-0.4426^{*}$ & -0.1203 & 0.1310 & 0.1846 & & & & & & & & & & & & \\
\hline LL & 0.0572 & $0.5487 *$ & -0.0572 & $0.3546^{*}$ & 0.3167 & $0.5373^{*}$ & -0.0113 & $0.3584 *$ & 0.1298 & $0.6172 *$ & $0.7836 *$ & $\mid-0.3034$ & & & & & & & & & & & \\
\hline LW & $0.4323 *$ & -0.1097 & 0.2583 & 0.1649 & $0.4055^{*}$ & 0.2566 & $0.5872 * 0$ & $0.3416^{*}$ & $-0.3639^{*}$ & $0.4110^{*}$ & 0.1859 & -0.3387 & -0.0998 & & & & & & & & & & \\
\hline LA & $\mid-0.0988$ & $0.5709^{*}$ & 0.0384 & $0.4181^{*}$ & $0.4586^{*}$ & $0.6219^{*}$ & $|0.2168|$ & $0.4765^{*}$ & -0.0118 & $0.4380^{*}$ & $0.6828^{*}$ & $-0.4192^{*}$ & $0.9250^{*}$ & 0.2848 & & & & & & & & & \\
\hline FLW & -0.1776 & 0.0755 & 0.2038 & 0.4141* & 0.1044 & 0.0602 & 0.1902 & 0.1690 & 0.3791 & -0.0838 & -0.2435 & $-0.3787^{*}$ & 0.2378 & -0.1561 & 0.1826 & & & & & & & & \\
\hline FSW & 0.2286 & 0.0874 & -0.0975 & $0.8117^{*}$ & $0.7284 *$ & $0.7186^{*}$ & -0.1976 & $0.6657 *$ & 0.1219 & $0.4128^{*}$ & 0.3056 & $0.7602^{*}$ & $0.5195^{*}$ & $0.3838^{*}$ & 0.6544* & $-0.3962 *$ & & & & & & & \\
\hline $\mathrm{FW}$ & -0.3168 & 0.3892 & 0.2256 & $0.5522^{*}$ & -0.2800 & $0.5444 *$ & -0.3062 & -0.3209 & 0.1814 & $0.3695^{*}$ & 0.2475 & -0.0049 & $-0.4342 *$ & 0.2129 & $-0.3496^{*}$ & -0.2250 & $0.4368^{*}$ & & & & & & \\
\hline SPF & $0.3357^{*}$ & -0.2624 & -0.2190 & 0.0261 & $0.6072^{*}$ & 0.2327 & -0.3290 & 0.0701 & $-0.5112^{*}$ & -0.0825 & -0.2852 & $0.6372^{*}$ & -0.0504 & -0.2489 & -0.1388 & -0.2299 & $0.3457^{*}$ & -0.0509 & & & & & \\
\hline $\mathrm{HSW}$ & -0.2110 & -0.0802 & 0.0189 & 0.2805 & 0.2698 & 0.2135 & -0.2069 & 0.1428 & 0.1302 & $0.4799^{*}$ & -0.2091 & $-0.4771^{*}$ & 0.3224 & 0.3372 & $0.4300^{*}$ & -0.0049 & $-0.5089^{*}$ & 0.2117 & -0.1950 & & & & \\
\hline SA & -0.2123 & -0.0176 & $0.4387 *$ & $-0.3649 *$ & $-0.3544 *$ & $-0.3596 *$ & -0.2203 & $\mid-0.1311$ & 0.0090 & $0.4723 *$ & $0.5642^{*}$ & 0.2387 & $-0.3503^{*}$ & -0.1822 & $-0.4146^{*}$ & $-0.4584^{*}$ & $0.5161^{*}$ & $0.3473^{*}$ & -0.0084 & -0.3245 & & & \\
\hline SW & -0.1780 & $0.5446 *$ & 0.1105 & 0.1083 & $0.2293^{*}$ & -0.0731 & -0.0224 & 0.1543 & -0.1874 & 0.1312 & $0.3928 *$ & \begin{tabular}{|c|} 
\\
\end{tabular} & $-0.4553^{*}$ & -0.3195 & $-0.5608^{*}$ & 0.0272 & -0.0047 & -0.2391 & -0.2006 & $-0.3778^{*}$ & 0.2548 & & \\
\hline $\mathrm{ABA}$ & $0.4873^{*}$ & $0.5678^{*}$ & $0.4295^{*}$ & $0.6059^{*}$ & $0.6105^{*}$ & $0.5155^{*}$ & 0.1939 & $0.6303 *$ & $0.6055^{*}$ & 0.0500 & -0.1248 & \begin{tabular}{|c|}
-0.1071 \\
\end{tabular} & 0.2178 & $0.4029^{*}$ & $0.3521^{*}$ & -0.1509 & $0.3672^{*}$ & -0.0245 & $0.4402 *$ & $0.4521^{*}$ & -0.0221 & $0.5046^{*}$ & \\
\hline SYP & $0.4686^{*}$ & $0.3733^{*}$ & $-0.2753 \mid$ & -0.2215 & 0.3062 & $-0.3579 *$ & $|-0.1311|$ & $-0.3978 *$ & $0.3493 *$ & $\mid-0.0664$ & $0.3580^{*}$ & -0.0343 & -0.1842 & -0.1173 & -0.2242 & $-0.4212^{*}$ & 0.1257 & 0.0152 & $-0.4284^{*}$ & -0.0631 & $0.3865^{*}$ & $0.3295^{*}$ & $0.5632^{*}$ \\
\hline
\end{tabular}

APPLIED ECOLOGY AND ENVIRONMENTAL RESEARCH 17(4): 7311-7332.

http://www.aloki.hu • ISSN 15891623 (Print) • ISSN 17850037 (Online)

DOI: http://dx.doi.org/10.15666/aeer/1704_73117332

(c) 2019, ALÖKI Kft., Budapest, Hungary 
Table 3. Stepwise regression analysis for various traits of petunia for seed yield

\begin{tabular}{|c|c|c|c|c|c|c|}
\hline & Traits & Coefficients B & Standard error & $t$ Stat & Cumulative $R^{2}$ & Partial $R^{2} \%$ \\
\hline $\mathrm{X}_{1}$ & Photosynthetic rate & 0.224 & 0.213 & 1.053 & 0.403 & 40.30 \\
\hline $\mathrm{X}_{2}$ & Leaf temperature & -0.235 & 0.176 & -1.330 & 0.315 & 31.50 \\
\hline $\mathrm{X}_{3}$ & Chlorphyll a & 2.775 & 2.329 & 1.192 & 0.356 & 35.60 \\
\hline $\mathrm{X}_{4}$ & Chlorphyll b & 2.104 & 1.920 & 1.096 & 0.387 & 38.70 \\
\hline $\mathrm{X}_{5}$ & Stomata conductance & 120.311 & 47.385 & 2.539 & -0.126 & 12.60 \\
\hline $\mathrm{X}_{6}$ & Transpiration rate & -4.418 & 3.471 & -1.273 & 0.331 & 33.10 \\
\hline $\mathrm{X}_{7}$ & Sub-stomata $\mathrm{CO}_{2}$ concentration & -0.007 & 0.004 & -1.689 & -0.233 & 23.30 \\
\hline $\mathrm{X}_{8}$ & Water use efficiency & -0.070 & 0.364 & -0.191 & 0.866 & 86.60 \\
\hline $\mathrm{X}_{9}$ & Leaves per plant & -0.252 & 0.157 & -1.607 & 0.249 & 24.90 \\
\hline $\mathrm{X}_{10}$ & Plant height & 0.116 & 0.088 & 1.325 & 0.277 & 27.70 \\
\hline $\mathrm{X}_{11}$ & Stem diameter & 0.004 & 0.0627 & 0.068 & 0.950 & 95.00 \\
\hline $\mathrm{X}_{12}$ & Flowers per plant & 2.484 & 8.886 & 0.279 & 0.798 & 79.80 \\
\hline $\mathrm{X}_{13}$ & Leaf length & -0.115 & 0.096 & -1.203 & -0.315 & 31.50 \\
\hline $\mathrm{X}_{14}$ & Leaf width & -17.234 & 17.825 & -0.967 & 0.405 & 40.50 \\
\hline $\mathrm{X}_{15}$ & Leaf area & -80.073 & 80.280 & -0.997 & -0.392 & 39.20 \\
\hline $\mathrm{X}_{16}$ & Fresh leaf weight & 11.364 & 11.769 & 0.966 & 0.406 & 40.60 \\
\hline $\mathrm{X}_{17}$ & Fresh stem weight & -10.121 & 5.151 & -1.965 & -0.144 & 14.40 \\
\hline $\mathrm{X}_{18}$ & Flower weight & 0.068 & 0.1356 & 0.501 & 0.643 & 64.30 \\
\hline $\mathrm{X}_{19}$ & Seeds per fruit & -12.101 & 10.544 & -1.148 & -0.315 & 31.50 \\
\hline $\mathrm{X}_{20}$ & 100 -seed weight & -0.033 & 0.0589 & -0.563 & 0.604 & 60.40 \\
\hline $\mathrm{X}_{21}$ & Seed area & 7.062 & 4.326 & 1.632 & -0.178 & 17.80 \\
\hline $\mathrm{X}_{22}$ & Capsule weight & 31.497 & 18.527 & 1.701 & 0.164 & 16.40 \\
\hline $\mathrm{X}_{23}$ & Abscisic acid & 15.356 & 30.458 & 0.504 & 0.641 & 64.10 \\
\hline
\end{tabular}

Intercept $=128.387, \mathrm{R}^{2}=0.832$, Adjust $\mathrm{R}^{2}=0.276$, Standard Error $=0.728$

We performed principle component analysis to investigate the traits contributing higher towards seed yield per plant in petunia, four PCA (Table 4) were estimated which diverse variation among the studied traits. The PC1, PC2, PC3 and PC4 contributed variations of $29.60 \%, 16.60 \%, 13.90 \%$ and $10.5 \%$ while their cumulative proportion was $29.6 \%, 46.20 \%, 60.10 \%$ and $70.60 \%$ respectively. PC1 and PC2 contributed higher variation for respective studied traits (Fig. 3a) the eigenvalues of PCs was higher than 1 (Fig. 3b). Through the use of principle component analysis, principle factor analysis was performed to check the traits that were directly and highly associated with the seed yield per plant in petunia. The highly contributing factor traits from factor 1 which contributes $36.20 \%$ in total variation were chlorophyll a, chlorophyll b, stomata conductance, transpiration rate, water use efficiency, leaves per plant, stem diameter, leaf length, leaf area, seed yield per plant and abscisic acid (Table 5). Fawad et al. (2017) and Ali et al. (2014) suggested that the selection of genotypes on the basis of factor analysis (traits from factor 1) may be helpful to develop higher yield synthetic varieties and hybrids. The traits in factor 2 (from factor loading Table 5) showed that the selection on the basis of these traits will not be useful as segregation takes place in the next generation. The higher performance of petunia genotypes for chlorophyll $\mathrm{a}$, chlorophyll $\mathrm{b}$, stomata conductance, transpiration rate, water use efficiency, leaves per plant, stem diameter, leaf length, leaf area, seed yield per plant and abscisic acid indicated that the accumulation of organic compounds will be higher in the plant body. The accumulation of biomass in plant body is essential for 
the proper and enhanced growth and development of petunia (Huang, 2007; Huang and Yeh, 2009; Ali et al., 2011; Munir et al., 2016), the accumulation is generally in the leaf, stem and flowering parts of the plant body. Our findings are well supported by Filipovic et al. (2014) who demonstrated the role of factor analysis for effective selection criteria in maize breeding program. For understanding of the association among the petunia lines, we performed cluster analysis (Mahmood et al., 2016; Khorasani et al., 2011; Mostafavi et al., 2011; Ali et al. 2015, 2016). The results from clustering showed that the petunia lines IAGS-P8 and IAGS-P9 were highly associated with each other as compared with all other petunia lines (Fig. 4a) the association was verified through the development of minimum spanning tree (Fig. $4 b$ ) that showed very less distance between petunia lines IAGS-P8 and IAGS-P9 through the use of eigen values. The results showed that the petunia lines IAGS-P8 and IAGS-P9 may be used as two separate male or female lines to develop petunia hybrids. The petunia line IAGSP11 performed better for almost all studied traits, it may be used as male to develop good quality petunia hybrids. It also indicated that in future breeding program of IAGSP8, IAGS-P9 and IAGS-P11, these traits are important for primary selection to increase seed yield per plant of petunia under various environmental regimes. Moreover, this method proved to be more efficient as it reduced the cost, money, time and efficacy for better selection in petunia improvement program because the hybridization procedure of petunia is very difficult. When researcher take important traits like seed yield and flower size in account then there may be a chance to improve yield and quality of petunia. However, further studies are required which should cover different years and locations.

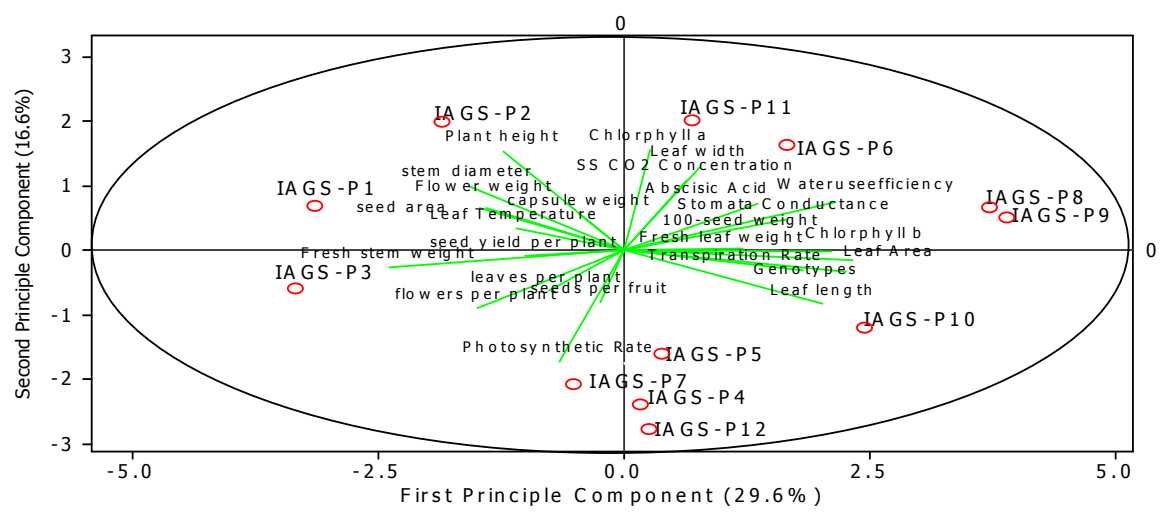

a

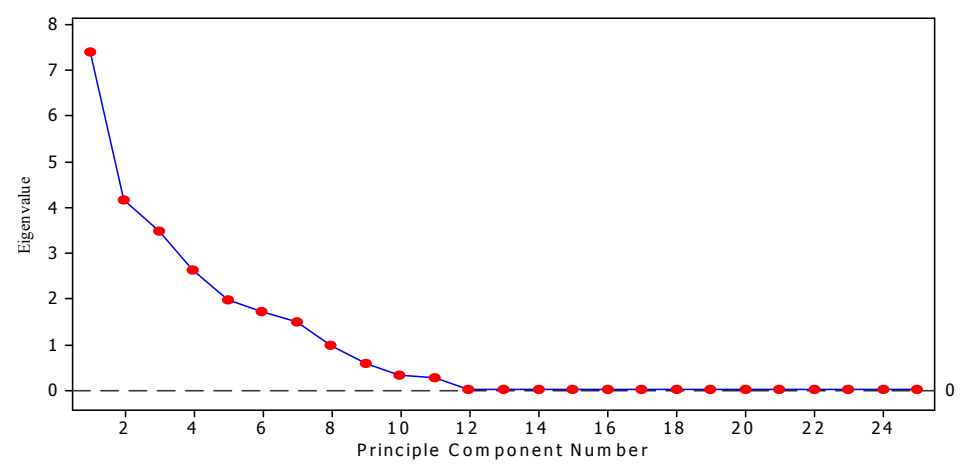

b

Figure 3. a Principle component analysis of yield and its attributing traits. $\boldsymbol{b}$ Scree plot and respective eigen values 
Table 4. Principal component analysis

\begin{tabular}{c|c|c|c|c}
\hline Eigen value & 7.3977 & 4.1516 & 3.4767 & 2.6343 \\
\hline Proportion & 0.296 & 0.166 & 0.139 & 0.105 \\
\hline Cumulative & 0.296 & 0.462 & 0.601 & 0.706 \\
\hline Traits & PC1 & PC2 & PC3 & PC4 \\
\hline Photosynthetic rate & 0.689 & 0.415 & 0.016 & 0.055 \\
\hline Leaf temperature & -0.15 & 0.083 & -0.336 & -0.093 \\
\hline Chlorphyll a & 0.035 & 0.376 & 0.07 & 0.036 \\
\hline Chlorphyll b & 0.286 & -0.005 & -0.025 & -0.217 \\
\hline Stomata conductance & 0.221 & 0.118 & -0.297 & -0.047 \\
\hline Transpiration rate & 0.313 & -0.037 & 0.151 & -0.162 \\
\hline Sub-stomata CO2 concentration & 0.087 & 0.263 & 0.029 & 0.002 \\
\hline Water use efficiency & 0.29 & 0.183 & 0.123 & -0.202 \\
\hline Leaves per plant & -0.104 & -0.15 & -0.34 & 0.257 \\
\hline Plant height & -0.166 & 0.371 & 0.137 & -0.033 \\
\hline Stem diameter & -0.213 & 0.243 & -0.044 & -0.124 \\
\hline Flowers per plant & -0.203 & -0.216 & 0.315 & -0.108 \\
\hline Leaf length & 0.274 & -0.201 & -0.01 & 0.158 \\
\hline Leaf width & 0.104 & 0.313 & 0.076 & 0.248 \\
\hline Leaf area & 0.305 & -0.077 & 0.018 & 0.241 \\
\hline Fresh leaf weight & 0.136 & -0.007 & -0.185 & -0.041 \\
\hline Fresh stem weight & -0.324 & -0.064 & 0.176 & 0.002 \\
\hline Flower weight & -0.191 & 0.16 & 0.018 & 0.337 \\
\hline Seeds per fruit & -0.032 & -0.196 & 0.414 & -0.134 \\
\hline 100-seed weight & 0.161 & 0.009 & -0.074 & 0.371 \\
\hline Seed area & -0.199 & 0.162 & 0.082 & -0.106 \\
\hline Seed yield per plant & -0.136 & -0.021 & -0.316 & -0.056 \\
\hline Capsule weight & -0.062 & 0.135 & -0.205 & -0.499 \\
\hline Abscisic acid & 0.184 & 0.178 & 0.336 & 0.075 \\
\hline & & & & \\
\hline & &
\end{tabular}

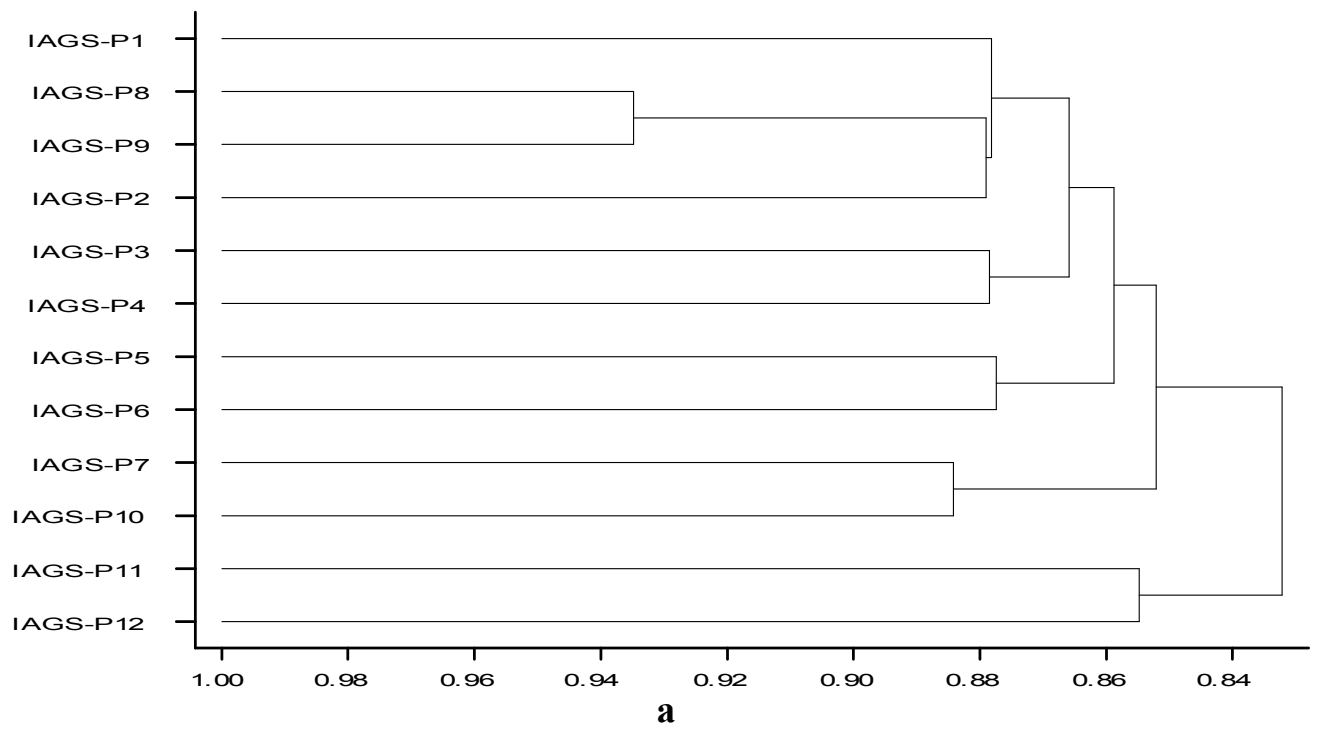




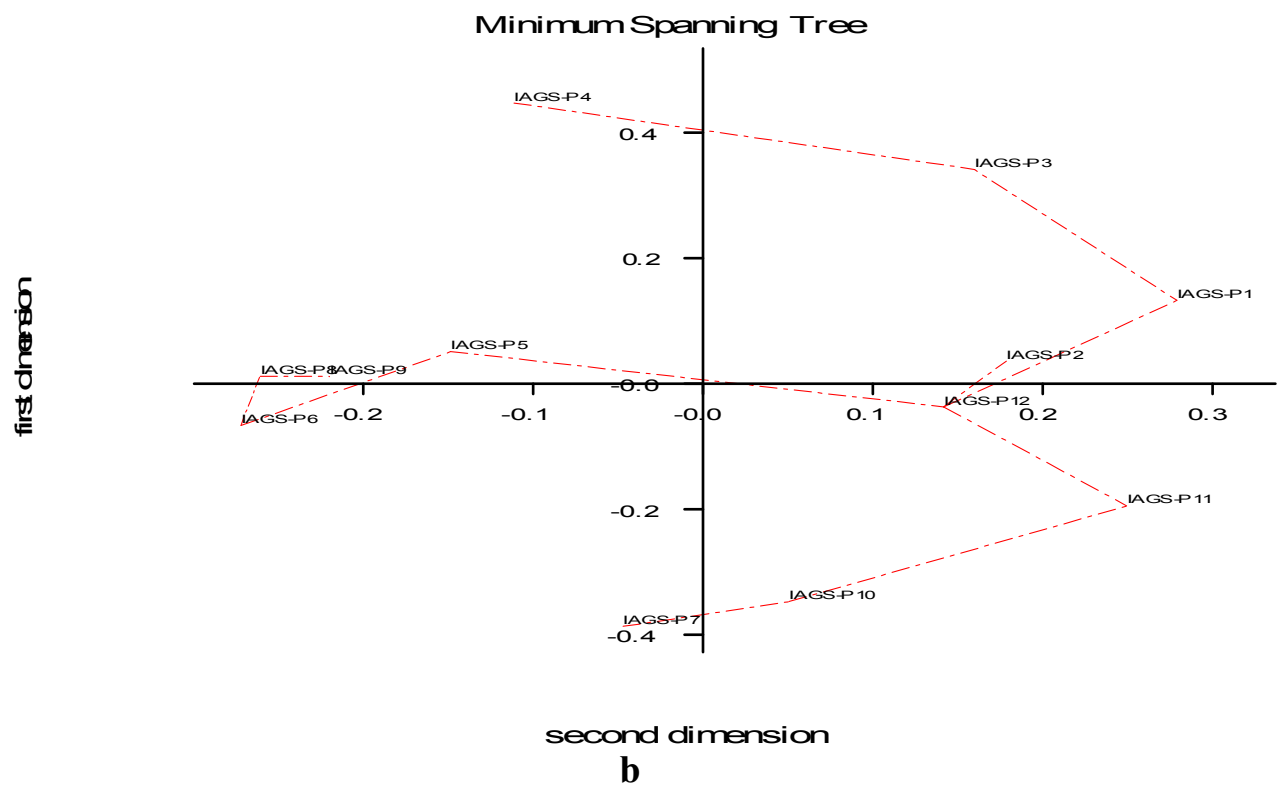

Figure 4. a Dendrogram analysis based on hierarchal clustering. Association of petunia lines on genetic basis of all studied traits. $\boldsymbol{b}$ Minimum spanning tree using egine values for petunia lines on the basis of all studied traits

Table 5. Factor loadings for various traits of petunia

\begin{tabular}{|c|c|c|}
\hline Factor1 & Factor loadings & \% Communality \\
\hline $\begin{array}{c}\text { Chlorophyll a } \\
\text { Chlorophyll b } \\
\text { Stomata conductance } \\
\text { Transpiration rate } \\
\text { Water use efficiency } \\
\text { leaves per plant } \\
\text { stem diameter } \\
\text { Leaf length } \\
\text { Leaf area } \\
\text { Seed yield per plant } \\
\text { Abscisic acid } \\
\text { Number of flowers per plant }\end{array}$ & $\begin{array}{l}0.603 \\
0.766 \\
0.564 \\
0.873 \\
0.970 \\
0.659 \\
0.929 \\
0.887 \\
0.850 \\
0.867 \\
0.836 \\
0.756\end{array}$ & 36.2 \\
\hline \multicolumn{3}{|l|}{ Factor2 } \\
\hline $\begin{array}{c}\text { Sub-stomata CO2 concentration } \\
\text { Plant height } \\
\text { Fresh shoot weight }\end{array}$ & $\begin{array}{l}-0.545 \\
-0.894 \\
-0.628\end{array}$ & 27.1 \\
\hline \multicolumn{3}{|l|}{ Factor3 } \\
\hline $\begin{array}{c}\text { Seed size } \\
\text { Leaf length } \\
\text { Leaf area } \\
\text { Fresh leaf weight } \\
\text { Seed per fruit }\end{array}$ & $\begin{array}{l}0.309 \\
0.333 \\
0.307 \\
0.369 \\
0.354\end{array}$ & 13.1 \\
\hline \multicolumn{3}{|l|}{ Factor4 } \\
\hline $\begin{array}{l}\text { Photosynthetic rate } \\
\text { Capsule weight } \\
\text { Leaf width } \\
\text { stem diameter } \\
\text { Leaf temperature } \\
\text { Flower weight } \\
\text { 100-seed weight }\end{array}$ & $\begin{array}{l}0.211 \\
0.116 \\
0.129 \\
0.279 \\
0.217 \\
0.163 \\
0.296\end{array}$ & 9.40 \\
\hline Cumulative variance & & 85.80 \\
\hline
\end{tabular}




\section{Conclusion}

The present study was carried out to investigate the higher seed yield petunia line during the experimental seasons of 2011-2014. The data for various morphological, physiological and seed yield traits were recorded, analyzed and interpreted for final inferences. It was found that the lines IAGS-P8, IAGS-P9 and IAGS-P11 performed well. From correlation analysis, heritability, genetic advance, principle component analysis, factor analysis and regression analysis that stomata conductance, chlorophyll ' $a$ ' contents, chlorophyll ' $b$ ' contents, flowers per plant, flower fresh weight, seed area, seed weight and abscisic acid contributed higher to seed yield per plant in petunia. The higher abscisic acid contribution suggested that the petunia genotypes may be used to develop good seed yield per plant and large number of flowers per plant in petunia. The more research and experimentation should be carried out to improve yield and quality of petunia flower.

Conflict of interests. The authors declare the absence of potential conflict of interests.

\section{REFERENCES}

[1] Ahmad, A., Tahir, M. (2017): Role of Zinc Sulphate for Maize (Zea mays L.) and Mungbean (Vigna radiata L. Wilczek) yield improvement: a review. - Bulletin of Biological and Allied Sciences Research 2: 1-17.

[2] Ahmad, H. M., Ahsan, M., Ali, Q., Javed, I. (2012): Genetic variability, heritability and correlation studies of various quantitative traits of mungbean (Vigna radiate L.) at different radiation levels. - International Research Journal of Microbiology 3(11): 352362.

[3] Ali, F., Kanwal, N., Ahsan, M., Ali, Q., Bibi, I., Niazi, N. K. (2015): Multivariate analysis of grain yield and its attributing traits in different maize hybrids grown under heat and drought stress. - Scientifica. http://dx.doi.org/10.1155/2015/563869.

[4] Ali, Q., Ahsan, M., Hussain, B., Elahi, M., Khan, N. H., Ali, F., Elahi, F., Shahbaz, M., Ejaz, M., Naees, M. (2011): Genetic evaluation of maize (Zea mays L.) accessions under drought stress. - International Research Journal of Microbiology 11: 437-441.

[5] Ali, Q., Ahsan, M., Ali, F., Aslam, M., Khan, N. H., Munzoor, M., Mustafa, H. S. B., Muhammad, S. (2013): Heritability, heterosis and heterobeltiosis studies for morphological traits of maize (Zea mays L.) seedlings. - Advancements in Life Sciences 1(1): 52-63.

[6] Ali, Q., Ali, A., Ahsan, M., Ali, S., Khan, N. H., Muhammad, S., Abbas, H. G., Nasir, I. A., Husnain, T. (2014): Line $\times$ Tester analysis for morpho-physiological traits of Zea mays L. seedlings. - Advancements in Life Sciences 1(4): 242-253.

[7] Ali, Q., Ahsan, M., Malook, S., Kanwal, N., Ali, F., Ali, A., Ahmed, W., Ishfaq, M., Saleem, M. (2016): Screening for drought tolerance: comparison of maize hybrids under water deficit condition. - Advancements in Life Sciences 3(2): 51-58.

[8] Bala, M. (2012): Floriculture General and Special. - Partos Timisoara.

[9] Bender, S. (2006): New Types of Perfect Petunias. - Issue of Southern Living. www.southernliving.com/.../new-typesperfect.

[10] Blanchard, M., Runkle, E. (2009): Energy efficient annuals part 6: Petunias. Greenhouse Grower 27(9): 36-41.

[11] Burton, G. W. (1951): Quantitative Inheritance in pearl millet (Pennisetum glaucum L) 1. - Agronomy Journal 43(9): 409-417. 
[12] Dole, J., Wilkins, H. (1999): Floriculture Principles and Species. - Prentice Hall, Upper Saddle River, NJ.

[13] EL-Badawy, M. E. M., Mehasen, S. A. S. (2011): Multivariate analysis for yield and its components in maize under zinc and nitrogen fertilization levels. - Australian Journal of Basic and Applied Sciences 5: 3008-3015.

[14] Erwin, J. (2006): Flower Breeding and Genetics. Factor Affecting Flowering in Ornamental Plants. - In: Anderson, N. O. (ed.) Flower Breeding and Genetics: Issues, Challenges and Opportunities for the 21st Century. Springer Netherlands.

[15] Falconer, D. S. (1989): Introduction to Quantitative Genetics. 3rd Ed. - Longman Scientific \& Technical, Harlow.

[16] Fawad, A., Ahsan, M., Ali, Q., Kanwal, N. (2017): Phenotypic stability of Zea mays grain yield and its attributing traits under drought stress. - Frontiers in Plant Science 8: 1397.

[17] Filipovic, M., Babic, M., Delic, N., Bekavac, G., Babic, V. (2014): Determination of relevant breeding criteria by the path and factor analysis in maize. - Genetika 46: 41-49.

[18] Ganga, M., Jayalakshmi, S., Jegadeeswari, K., Padmadevi, K., Jawaharlal, M. (2011): Petunia. - In: Kole, C. (ed). Wild Crop Relatives: Genomic and Breeding Resources Plantation and Ornamental Crop. Springer, Berlin.

[19] Greenacre, M. (2010): Niplots in Practice. - BBVA Foundation, Madrid. www.multivariatestatistics.org.

[20] Griesbach, J. R. (2007): Petunia. - In: Anderson, N. O. (ed.) Flower Breeding and Genetics. - Springer, Netherlands, pp: 301-336.

[21] Hiscox, J. D., Israelstam, G. F. (1978): A method for the extraction of chlorophyll from leaf tissue without maceration. - Canadian Journal of Botany 57: 1332-1334.

[22] Huang, L. C. (2007): Behavioral differences in pre-purchase processes between purchasers of flowers for self use and for gift use. - Horticultural Technology 17: 183190.

[23] Huang, L. C., Yeh, T. F. (2009): Floral consumption values for consumer groups with different purchase choices for flowers. - Horticulture Technology 19: 563-571.

[24] Kaczperski, M. P., Carlson, W. H., Karlsson, M. G. (1991): Growth and development of Petunia. hybrids as a function of temperature and irradiance. - Journal of American Society of Horticultural Sciences 116: 232-237.

[25] Khorasani, S. K., Mostafavi, K., Zandipour, E., Heidarian, K. (2011): Multivariate analysis of agronomic traits of new corn hybrids (Zea mays L.). - International Journal of Agricultural Sciences 1: 314-322.

[26] Mahmood, A., Haider, M. S., Ali, Q., Nasir, I. A. (2017): Multivariate analysis to assess abscisic acid content association with different physiological and plant growth related traits of Petunia. - Acta Agriculturae Slovenica 109(2): 175-186.

[27] Mahmood, Z., Afzal, M., Ahmad, M., Munir, M. A., Ali, M. I., Sharif, M. N., Maqbool, R. (2016): Genetic analysis for morphological traits of Euphorbia helioscopia. - Bulletin of Biological and Allied Sciences Research 1: 5.

[28] Mostafavi, K., Shoahosseini, M., Geive, H. S. (2011): Multivariate analysis of variation among traits of corn hybrids traits under drought stress. - International Journal of Agricultural Sciences 1: 416-422.

[29] Munir, M. A., Ahmad, M., Ali, M. I., Mahmood, Z., Afzal, M., Sharif, M. N., Aslam, M. (2016): Correlation and regression analysis of morphological traits in Rumex dentatus. Bulletin of Biological and Allied Sciences Research 1: 2.

[30] Mustafa, H. S. B., Tariq, M., Ahsan, U., Amjad, S., Abdul, N. B., Muhammad, N., Rahat, A. (2017): Role of seed priming to enhance growth and development of crop plants against biotic and abiotic stresses. - Bulletin of Biological and Allied Sciences Research 2: $1-11$.

[31] Sah, S. K., Reddy, K. R., Li, J. (2016): Abscisic acid and abiotic stress tolerance in crop plants. - Frontiers in Plant Science 7: 571. 
[32] Saradadevi, R., Palta, J. A., Siddique, K. H. (2017): ABA-mediated stomatal response in regulating water use during the development of terminal drought in wheat. - Frontiers in Plant Science 8: 1251.

[33] Selaru, E. (2008): Culture Flowers for Garden. - Ceres, Bucharest.

[34] Seo M and Koshiba T (2002): Complex regulation of ABA biosynthesis in plants. Trends in Plant Science 7(1): 41-8.

[35] Skubacz, A., Daszkowska-Golec, A., Szarejko, I. (2016): The role and regulation of ABI5 (ABA-Insensitive 5) in plant development, abiotic stress responses and phytohormone crosstalk. - Frontiers in Plant Science 7: 1884.

[36] Steel, R. G. D., Torrie, J. H., Dicky, D. A. (1997): Principles and Procedures of Statistics. A Biometrical Approach. 3rd Ed. - McGraw Hill, New York, pp: 400-428.

[37] Stehmann, J. R., Forzza, R. C., Salino, A., Sobral, M., da Costa, D. P., Kamino, L. H. (eds.) Plantas, da floresta Atlântica. - Jardim Botânico do Rio de Janeiro, Rio de Janeiro.

[38] Stuurman, J., Hoballah, M. E., Broger, L., Moore, J., Basten, C., Kuhlemeier, C. (2004): Dissection of floral pollination syndromes in Petunia. - Genetics 168: 1585-1599.

[39] Toma, F. (2009): Floriculture and Floral Art. - INVEL Multimedia, Bucharest.

[40] Toma, F., Vasilescu, T., Petra, S., Zamfir-Vasca, D. (2011): Research concerning the propagation by cutting of some new cultivars of petunia. - USAMV Bucharest Seria B 55: 253-256.

[41] Zhao, Y., Wang, Y., Yang, H., Wang, W., Wu, J., Hu, X. (2016): Quantitative proteomic analyses identify ABA-related proteins and signal pathways in maize leaves under drought conditions. - Frontiers in Plant Science 7: 1827.

\section{APPENDIX}

\section{Photosynthetic rate}

Genotypes mean homogeneous groups

IAGS-P11 $19.578 \mathrm{~A}$

IAGS-P8 19.267 B

IAGS-P9 $18.245 \mathrm{C}$

IAGS-P7 16.888 D

IAGS-P4 16.102 E

IAGS-P5 $15.334 \mathrm{~F}$

IAGS-P3 $13.572 \mathrm{G}$

IAGS-P10 $11.245 \mathrm{H}$

IAGS-P1 11.112 I

IAGS-P6 $9.9033 \mathrm{~J}$

IAGS-P2 $9.1093 \mathrm{~K}$

IAGS-P12 8.8883 L

Alpha 0.05 Standard Error for Comparison 9.393E-03

\section{Leaf temperature}

Genotypes mean homogeneous groups

IAGS-P11 24.620 A

IAGS-P8 $24.175 \mathrm{~B}$

IAGS-P9 23.835 C

IAGS-P3 $23.730 \mathrm{C}$

IAGS-P1 22.176 D

IAGS-P2 21.428 E 
IAGS-P4 21.166 F

IAGS-P10 19.288 G

IAGS-P5 $18.951 \mathrm{H}$

IAGS-P12 $18.930 \mathrm{H}$

IAGS-P7 18.288 I

IAGS-P6 17.732 J

Alpha 0.05 Standard Error for Comparison 0.0613

\section{Chlorphyll a}

Genotypes mean homogeneous groups

IAGS-P11 $3.5467 \mathrm{~A}$

IAGS-P12 3.5267 AB

IAGS-P9 3.5167 AB

IAGS-P8 $3.4267 \mathrm{~B}$

IAGS-P7 $3.1867 \mathrm{C}$

IAGS-P2 $3.1667 \mathrm{CD}$

IAGS-P3 3.0867 CDE

IAGS-P1 $3.0867 \mathrm{CDE}$

IAGS-P4 3.0567 DE

IAGS-P5 $3.0367 \mathrm{E}$

IAGS-P10 3.0067 E

IAGS-P6 2.7467 F

Alpha 0.05 Standard Error for Comparison 0.0579

4. Chlorphyll b

Genotypes mean homogeneous groups

IAGS-P11 $1.9267 \mathrm{~A}$

IAGS-P9 $1.9067 \mathrm{~A}$

IAGS-P8 $1.9067 \mathrm{~A}$

IAGS-P6 1.8167 B

IAGS-P10 1.7567 B

IAGS-P12 $1.5967 \mathrm{C}$

IAGS-P2 $1.4167 \mathrm{D}$

IAGS-P4 $1.4067 \mathrm{D}$

IAGS-P1 $1.3667 \mathrm{DE}$

IAGS-P5 $1.3067 \mathrm{EF}$

IAGS-P7 $1.2667 \mathrm{~F}$

IAGS-P3 $1.2567 \mathrm{~F}$

Alpha 0.05 Standard Error for Comparison 0.0386

\section{Stomata conductance}

Genotypes mean homogeneous groups

IAGS-P11 $0.0567 \mathrm{~A}$

IAGS-P7 $0.0433 \mathrm{AB}$

IAGS-P9 $0.0400 \mathrm{BC}$

IAGS-P8 $0.0367 \mathrm{BCD}$

IAGS-P10 0.0367 BCD

IAGS-P1 $0.0267 \mathrm{CDE}$

IAGS-P12 0.0267 CDE

IAGS-P6 0.0267 CDE 
IAGS-P3 0.0233 DE

IAGS-P2 $0.0200 \mathrm{E}$

IAGS-P4 $0.0167 \mathrm{E}$

IAGS-P5 0.0133 E

Alpha 0.05 Standard Error for Comparison 6.804E-03

6. Transpiration rate

Genotypes mean homogeneous groups

IAGS-P10 $1.4567 \mathrm{~A}$

IAGS-P11 $1.4500 \mathrm{~A}$

IAGS-P8 1.3933 B

IAGS-P9 $1.2500 \mathrm{C}$

IAGS-P5 $0.8633 \mathrm{D}$

IAGS-P1 $0.8267 \mathrm{E}$

IAGS-P12 $0.7733 \mathrm{~F}$

IAGS-P7 $0.7700 \mathrm{~F}$

IAGS-P6 $0.6833 \mathrm{G}$

IAGS-P2 $0.5233 \mathrm{H}$

IAGS-P4 $0.5167 \mathrm{H}$

IAGS-P3 0.0967 I

Alpha 0.05 Standard Error for Comparison 0.0176

7. Sub-stomata $\mathrm{CO}_{2}$ concentration

Genotypes mean homogeneous groups

IAGS-P11 255.00 A

IAGS-P8 247.33 B

IAGS-P12 237.00 C

IAGS-P9 205.33 D

IAGS-P10 $181.00 \mathrm{E}$

IAGS-P3 $174.00 \mathrm{~F}$

IAGS-P1 $148.00 \mathrm{G}$

IAGS-P2 $143.33 \mathrm{H}$

IAGS-P4 68.667 I

IAGS-P6 66.000 J

IAGS-P5 $33.333 \mathrm{~K}$

IAGS-P7 27.667 L

Alpha 0.05 Standard Error for Comparison 0.8165

8. Water use efficiency

Genotypes mean homogeneous groups

IAGS-P11 $14.641 \mathrm{~A}$

IAGS-P8 $12.953 \mathrm{~B}$

IAGS-P9 8.7000 C

IAGS-P7 8.2507 D

IAGS-P1 7.4390 E

IAGS-P6 6.3847 F

IAGS-P2 5.7450 G

IAGS-P10 5.0213 H

IAGS-P5 $4.7317 \mathrm{H}$

IAGS-P12 4.2437 I 
IAGS-P4 2.6817 J

IAGS-P3 $0.7123 \mathrm{~K}$

Alpha 0.05 Standard Error for Comparison 0.1471

9. Leaves per plant

Genotypes mean homogeneous groups

IAGS-P9 91.333 A

IAGS-P11 91.333 A

IAGS-P8 90.333 B

IAGS-P2 $89.333 \mathrm{C}$

IAGS-P7 $88.333 \mathrm{D}$

IAGS-P4 87.333 E

IAGS-P12 86.333 F

IAGS-P6 $85.333 \mathrm{G}$

IAGS-P5 $84.333 \mathrm{H}$

IAGS-P3 83.333 I

IAGS-P10 82.333 J

IAGS-P1 $81.333 \mathrm{~K}$

Alpha 0.05 Standard Error for Comparison 0.4714

10. Plant height

Genotypes mean homogeneous groups

IAGS-P11 $62.357 \mathrm{~A}$

IAGS-P9 62.247 A

IAGS-P8 61.127 B

IAGS-P3 60.247 C

IAGS-P4 59.997 D

IAGS-P12 $59.347 \mathrm{E}$

IAGS-P5 56.797 F

IAGS-P7 $52.367 \mathrm{G}$

IAGS-P10 $51.947 \mathrm{H}$

IAGS-P6 49.017 I

IAGS-P1 $47.357 \mathrm{~J}$

IAGS-P2 $47.017 \mathrm{~K}$

Alpha 0.05 Standard Error for Comparison 0.0910

11. Stem diameter

Genotypes mean homogeneous groups

IAGS-P11 $0.5967 \mathrm{~A}$

IAGS-P9 $0.5733 \mathrm{~B}$

IAGS-P8 $0.5507 \mathrm{C}$

IAGS-P3 $0.5480 \mathrm{D}$

IAGS-P6 0.5210 E

IAGS-P4 $0.5120 \mathrm{~F}$

IAGS-P2 $0.5047 \mathrm{G}$

IAGS-P7 $0.4930 \mathrm{H}$

IAGS-P12 $0.4920 \mathrm{H}$

IAGS-P5 $0.4550 \mathrm{I}$

IAGS-P10 0.4433 J

IAGS-P1 $0.4420 \mathrm{~J}$ 
Alpha 0.05 Standard Error for Comparison 1.217E-03

12. Flowers per plant

Genotypes mean homogeneous groups

IAGS-P11 147.33 A

IAGS-P9 $146.33 \mathrm{~B}$

IAGS-P8 145.33 C

IAGS-P12 $141.33 \mathrm{D}$

IAGS-P3 141.33 D

IAGS-P1 140.33 E

IAGS-P6 139.33 F

IAGS-P2 $139.33 \mathrm{~F}$

IAGS-P4 139.33 F

IAGS-P5 $138.33 \mathrm{G}$

IAGS-P7 $137.33 \mathrm{H}$

IAGS-P10 136.33 I

Alpha 0.05 Standard Error for Comparison 0.4714

13. Leaf length

Genotypes mean homogeneous groups

IAGS-P8 7.000 A

IAGS-P11 6.9100 B

IAGS-P12 6.3400 C

IAGS-P9 6.1500 D

IAGS-P5 6.1000 E

IAGS-P6 6.0900 E

IAGS-P7 6.0400 F

IAGS-P2 $5.8900 \mathrm{G}$

IAGS-P10 5.8900 G

IAGS-P4 5.2100 H

IAGS-P1 $5.1600 \mathrm{I}$

IAGS-P3 5.1300 I

Alpha 0.05 Standard Error for Comparison 0.0216

\section{Leaf width}

Genotypes mean homogeneous groups

IAGS-P11 $1.1033 \mathrm{~A}$

IAGS-P8 $1.1033 \mathrm{~A}$

IAGS-P9 $1.1033 \mathrm{~A}$

IAGS-P10 1.0833 AB

IAGS-P3 $1.0633 \mathrm{ABC}$

IAGS-P1 $1.0433 \mathrm{BCD}$

IAGS-P6 1.0333 BCD

IAGS-P5 1.0133 CDE

IAGS-P12 1.0033 DEF

IAGS-P4 0.9733 EF

IAGS-P2 $0.9733 \mathrm{EF}$

IAGS-P7 $0.9533 \mathrm{~F}$

Alpha 0.05 Standard Error for Comparison 0.0249 


\section{Leaf area}

Genotypes mean homogeneous groups

IAGS-P11 7.423 A

IAGS-P8 $6.603 \mathrm{~B}$

IAGS-P9 6.107 C

IAGS-P12 5.847 CD

IAGS-P2 5.837 CDE

IAGS-P6 5.677 DE

IAGS-P7 5.667 DE

IAGS-P5 5.557 E

IAGS-P10 4.850 F

IAGS-P1 $4.703 \mathrm{~F}$

IAGS-P3 $4.413 \mathrm{G}$

IAGS-P4 $3.990 \mathrm{H}$

Alpha 0.05 Standard Error for Comparison 0.1392

16. Fresh leaf weight

Genotypes mean homogeneous groups

IAGS-P9 $0.7660 \mathrm{~A}$

IAGS-P11 0.7120 AB

IAGS-P8 0.7100 AB

IAGS-P10 0.6920 B

IAGS-P7 $0.6880 \mathrm{~B}$

IAGS-P12 0.6840 B

IAGS-P5 0.6680 BC

IAGS-P3 $0.6600 \mathrm{BC}$

IAGS-P4 $0.6220 \mathrm{CD}$

IAGS-P2 $0.5880 \mathrm{D}$

IAGS-P6 $0.5760 \mathrm{D}$

IAGS-P1 $0.4860 \mathrm{E}$

Alpha 0.05 Standard Error for Comparison 0.0294

17. Fresh stem weight

Genotypes mean homogeneous groups

IAGS-P8 $52.610 \mathrm{~A}$

IAGS-P11 51.960 B

IAGS-P9 51.190 C

IAGS-P1 $50.070 \mathrm{D}$

IAGS-P2 49.960 DE

IAGS-P12 49.940 E

IAGS-P6 $49.940 \mathrm{E}$

IAGS-P4 $49.940 \mathrm{E}$

IAGS-P3 $47.740 \mathrm{~F}$

IAGS-P5 $47.440 \mathrm{G}$

IAGS-P10 $45.410 \mathrm{H}$

IAGS-P7 44.490 I

Alpha 0.05 Standard Error for Comparison 0.0535

18. Flower weight 
Genotypes mean homogeneous groups

IAGS-P9 $0.6633 \mathrm{~A}$

IAGS-P11 $0.6233 \mathrm{AB}$

IAGS-P3 0.6143 BC

IAGS-P8 $0.6143 \mathrm{BC}$

IAGS-P4 $0.6053 \mathrm{BC}$

IAGS-P6 0.5963 BCD

IAGS-P2 0.5943 BCD

IAGS-P10 0.5923 BCD

IAGS-P1 0.5903 BCD

IAGS-P5 $0.5863 \mathrm{BCD}$

IAGS-P12 $0.5763 \mathrm{CD}$

IAGS-P7 0.5593 D

Alpha 0.05 Standard Error for Comparison 0.0205

19. Seeds per fruit

Genotypes mean homogeneous groups

IAGS-P11 872.33 A

IAGS-P9 $871.33 \mathrm{~B}$

IAGS-P8 870.33 C

IAGS-P1 $867.33 \mathrm{D}$

IAGS-P12 867.33 D

IAGS-P10 866.33 E

IAGS-P2 866.33 E

IAGS-P5 $865.33 \mathrm{~F}$

IAGS-P5 $864.33 \mathrm{G}$

IAGS-P4 $863.33 \mathrm{H}$

IAGS-P7 862.33 I

IAGS-P3 857.33 J

Alpha 0.05 Standard Error for Comparison 0.4714

20. 100-seed weight

Genotypes mean homogeneous groups

IAGS-P11 $12.130 \mathrm{~A}$

IAGS-P8 $12.120 \mathrm{AB}$

IAGS-P9 $12.110 \mathrm{BC}$

IAGS-P1 $12.100 \mathrm{CD}$

IAGS-P7 12.090 DE

IAGS-P10 $12.080 \mathrm{E}$

IAGS-P2 $12.080 \mathrm{E}$

IAGS-P12 $12.020 \mathrm{~F}$

IAGS-P3 $12.010 \mathrm{~F}$

IAGS-P6 $11.990 \mathrm{G}$

IAGS-P5 $11.970 \mathrm{H}$

IAGS-P4 11.890 I

Alpha 0.05 Standard Error for Comparison 8.165E-03

21. Seed area

Genotypes mean homogeneous groups 
IAGS-P11 $0.3753 \mathrm{~A}$

IAGS-P9 $0.3723 \mathrm{~A}$

IAGS-P8 $0.3693 \mathrm{AB}$

IAGS-P12 $0.3683 \mathrm{ABC}$

IAGS-P1 0.3653 ABCD

IAGS-P2 $0.3593 \mathrm{BCD}$

IAGS-P3 $0.3583 \mathrm{CD}$

IAGS-P6 0.3563 DE

IAGS-P4 0.3463 EF

IAGS-P7 $0.3433 \mathrm{~F}$

IAGS-P5 0.3393 FG

IAGS-P10 $0.3323 \mathrm{G}$

Alpha 0.05 Standard Error for Comparison 5.249E-03

22. Seed weight

Genotypes mean homogeneous groups

IAGS-P11 $116.20 \mathrm{~A}$

IAGS-P4 $115.98 \mathrm{~B}$

IAGS-P8 115.85 C

IAGS-P9 $115.40 \mathrm{D}$

IAGS-P12 115.32 D

IAGS-P7 115.32 D

IAGS-P6 115.31 D

IAGS-P10 115.06 E

IAGS-P1 $114.87 \mathrm{~F}$

IAGS-P2 $114.32 \mathrm{G}$

IAGS-P3 $114.26 \mathrm{G}$

IAGS-P5 $113.63 \mathrm{H}$

Alpha 0.05 Standard Error for Comparison 0.0618

23. Abscisic acid

Genotypes mean homogeneous groups

IAGS-P11 $0.1250 \mathrm{~A}$

IAGS-P8 0.1240 AB

IAGS-P9 $0.1240 \mathrm{AB}$

IAGS-P10 0.1230 AB

IAGS-P3 0.1230 AB

IAGS-P1 $0.1220 \mathrm{AB}$

IAGS-P12 0.1210 B

IAGS-P4 0.1140 C

IAGS-P5 $0.1110 \mathrm{CD}$

IAGS-P6 $0.1100 \mathrm{D}$

IAGS-P7 $0.1090 \mathrm{D}$

IAGS-P2 $0.1030 \mathrm{E}$

Alpha 0.05 Standard Error for Comparison 1.633E-03

24. Seed yield per plant

Genotypes mean homogeneous groups

IAGS-P11 54.153 A

IAGS-P9 53.353 B 
IAGS-P8 52.263 C

IAGS-P6 52.143 D

IAGS-P2 51.263 E

IAGS-P5 51.263 E

IAGS-P10 50.153 F

IAGS-P7 $49.593 \mathrm{G}$

IAGS-P1 $48.923 \mathrm{H}$

IAGS-P12 48.703 I

IAGS-P3 $46.263 \mathrm{~J}$

IAGS-P4 43.603 K

Alpha 0.05 Standard Error for Comparison 0.0249 